\title{
Narrowing the difficulty gap
}

\section{for the Celis-Dennis-Tapia problem}

Immanuel M. Bomze

ISOR, University of Vienna, Austria

Michael L. Overton

Courant Institute of Math. Sciences, New York University, U.S.A.

Dedicated to John E. Dennis Jr. and Richard A. Tapia

on the occasion of their 75th birthdays

October 9,2014

\begin{abstract}
We study the Celis-Dennis-Tapia (CDT) problem: minimize a non-convex quadratic function over the intersection of two ellipsoids. In contrast to the well-studied trust region problem where the feasible set is just one ellipsoid, the CDT problem is not yet fully understood. Our main objective in this paper is to narrow the difficulty gap that occurs when the Hessian of the Lagrangian is indefinite at all Karush-Kuhn-Tucker points. We prove new sufficient and necessary conditions both for local and global optimality, based on copositivity, giving a complete characterization in the degenerate case.
\end{abstract}

Key words: Copositive matrices, non-convex optimization, global optimality condition, polynomial optimization, trust region problem

October 9, 2014 


\section{Introduction}

We study the Celis-Dennis-Tapia (CDT) problem [CDT85]: minimize a nonconvex quadratic function over the intersection of two ellipsoids. This problem is a natural extension of the well-studied trust region problem CGT00. in which there is just one ellipsoidal constraint. Such problems arise quite naturally in iterative non-linear optimization procedures where in one iteration step, the objective and the constraints are approximated by quadratic models. However, while any trust region problem can be solved both in theory and in practice quite efficiently, the additional constraint makes the CDT problem substantially more challenging. Many articles have treated the analysis of this and related problems, including AZ09, BE06, BM14, BA13, CY99, CY09, GP05, JLL09, JL13, PY97, YB13, Yua90, YZ03.

Our main objective in this paper is to narrow the so-called difficulty gap. As long as the Hessian of the Lagrangian is positive semidefinite (psd) at some Karush-Kuhn-Tucker (KKT) point, methods similar to those developed for the trust region problem can be employed, so these cases are considered easy. However, it may happen that the Hessian of the Lagrangian is indefinite (throughout, we use this to mean "not psd") at all KKT points, and this phenomenon is what is usually meant by the "difficulty gap". In this paper, we prove new sufficient and necessary conditions for both global and local optimality, based on copositivity, giving a complete characterization in the degenerate case.

We denote vectors in $n$-dimensional Euclidean space $\mathbb{R}^{n}$ by bold-faced letters, the positive orthant by $\mathbb{R}_{+}^{n}$ and transposition by ${ }^{\top}$. We write $\mathrm{I}_{n}$ for the identity matrix of order $n$ and use $\mathrm{o}$ and $\mathrm{O}$ respectively for the zero vector and matrix, with their dimensions determined by the context. The Euclidean norm is denoted by $\|\cdot\|$.

An important property of symmetric matrices is that of copositivity. For a given cone $\Gamma \subset \mathbb{R}^{n}$, recall that a symmetric $n \times n$ matrix $\mathrm{S}$ is said to be $\Gamma$-copositive if and only if

$$
\mathrm{d}^{\top} \mathrm{Sd} \geq 0 \text { for all } \mathrm{d} \in \Gamma,
$$

i.e., $\mathrm{S}$ generates a quadratic form taking no negative values over the cone $\Gamma$. Therefore, any psd matrix $S$ is $\Gamma$-copositive, regardless of $\Gamma$, but not conversely. A matrix $\mathrm{S}$ is said to be strictly $\Gamma$-copositive if and only if

$$
d^{\top} S d>0 \text { for all } d \in \Gamma \backslash\{0\} .
$$

Any positive-definite matrix is strictly $\Gamma$-copositive, but again, not conversely. 
By scaling the constraints and making an affine transformation, we can reduce any $\mathrm{CDT}$ problem to the following form:

$$
z^{*}:=\min \left\{f(\mathrm{x}):\|\mathrm{x}\| \leq 1 \text { and }\left\|\mathrm{A}^{\top} \mathrm{x}-\mathrm{a}\right\| \leq 1\right\},
$$

with

$$
f(\mathrm{x})=\frac{1}{2} \mathrm{x}^{\top} \mathrm{Q} \mathrm{x}+\mathrm{q}^{\top} \mathrm{x}
$$

where $Q$ is a real symmetric matrix, with no assumptions on its inertia, $A$ is an $n \times m$ matrix, and $\mathrm{q} \in \mathbb{R}^{n}$, a $\in \mathbb{R}^{m}$. For simplicity, we assume that $\mathrm{A}$ has full row rank $n$, and to avoid the cases where the feasible set is empty or consists only of a single point, we assume that Slater's condition holds: there exists $\hat{x} \in \mathbb{R}^{n}$ such that

$$
\max \left\{\|\hat{x}\|,\left\|A^{\top} \hat{x}-a\right\|\right\}<1 .
$$

This can be checked by solving a convex trust region problem.

Consider the following two trust region problems:

$$
\left.\begin{array}{ll}
\min & \{f(\mathrm{x}):\|\mathrm{x}\| \leq 1\} \quad \text { and } \\
\min & \left\{f(\mathrm{x}):\left\|\mathrm{A}^{\top} \mathrm{x}-\mathrm{a}\right\| \leq 1\right\} .
\end{array}\right\}
$$

Any global solution to either of the trust region problems (5) that is also feasible for the other one constitutes a global solution to the CDT problem (2). Moreover, any local solution $\bar{x}$ to $(2)$ where at most one of the constraints is binding, i.e. which satisfies

$$
\min \left\{\|\bar{x}\|,\left\|A^{\top} \bar{x}-a\right\|\right\}<1,
$$

is necessarily a local solution to one of the trust region problems (5), and we know that there can be at most one local, non-global solution to a trust region problem [Mar94].

This leaves us with only one problematic region of the feasible set, namely

$$
B=\left\{\mathrm{x} \in \mathbb{R}^{n}:\|\mathrm{x}\|=1 \text { and }\left\|\mathrm{A}^{\top} \mathrm{x}-\mathrm{a}\right\|=1\right\}
$$

where both constraints are binding. We focus on this case in what follows.

It is convenient to square and scale the constraints in (2), replacing the CDT problem by

$$
z^{*}=\min \{f(\mathrm{x}): r(\mathrm{x}) \leq 0 \text { and } s(\mathrm{x}) \leq 0\},
$$

where

$$
\left.\begin{array}{l}
r(\mathrm{x})=\frac{1}{2}\left(\mathrm{x}^{\top} \mathrm{x}-1\right) \leq 0 \quad \text { and } \\
s(\mathrm{x})=\frac{1}{2}\left(\mathrm{x}^{\top} \mathrm{AA}^{\top} \mathrm{x}-2 \mathrm{a}^{\top} \mathrm{A}^{\top} \mathrm{x}+\|\mathrm{a}\|^{2}-1\right) \leq 0 .
\end{array}\right\}
$$


For later use, observe that the gradients of the objective (3) and constraints (9), evaluated at a given point $\bar{x}$, are

$$
\left.\begin{array}{rl}
\overline{\mathrm{g}} & :=\nabla f(\overline{\mathrm{x}})=\mathrm{Q} \overline{\mathrm{x}}+\mathrm{q}, \\
\overline{\mathrm{x}} & =\nabla r(\overline{\mathrm{x}}), \\
\overline{\mathrm{y}} & :=\nabla s(\overline{\mathrm{x}})=\mathrm{AA}^{\top} \overline{\mathrm{x}}-\mathrm{Aa} .
\end{array}\right\}
$$

We end this section by noting that whenever $\bar{x} \in B$ and the constraint gradients $\{\bar{x}, \bar{y}\}$ are linearly dependent, they necessarily are positively dependent in the sense that $\bar{y}=\alpha \bar{x}$ for some $\alpha>0$. This follows from the Slater condition (4), as convexity of the constraints and $r(\overline{\mathrm{x}})=s(\overline{\mathrm{x}})=0$ imply that for any strictly feasible point $\hat{x}$,

$$
\max \left\{(\hat{\mathrm{x}}-\overline{\mathrm{x}})^{\top} \overline{\mathrm{x}},(\hat{\mathrm{x}}-\overline{\mathrm{x}})^{\top} \overline{\mathrm{y}}\right\} \leq \max \{r(\hat{\mathrm{x}})-r(\overline{\mathrm{x}}), s(\hat{\mathrm{x}})-s(\overline{\mathrm{x}})\}<0 .
$$

This would be impossible if $\overline{\mathrm{y}}=\alpha \overline{\mathrm{x}}$ with $\alpha \leq 0$.

\section{Optimality Conditions}

\subsection{The polyhedral tangent cone and KKT points}

Let $\bar{x} \in \mathbb{R}^{n}$ be given, with $\bar{x}$ feasible for (8). We define the polyhedral tangent cone associated with $\bar{x}$ as the set of directions along which the binding constraints are feasible to first order, i.e.,

$$
\Gamma(\overline{\mathrm{x}}):=\left\{\begin{array}{ll}
\left\{\mathrm{d} \in \mathbb{R}^{n}: \overline{\mathrm{x}}^{\top} \mathrm{d} \leq 0 \text { and } \overline{\mathrm{y}}^{\top} \mathrm{d} \leq 0\right\} & \text { if } \overline{\mathrm{x}} \in B \\
\left\{\mathrm{~d} \in \mathbb{R}^{n}: \overline{\mathrm{x}}^{\top} \mathrm{d} \leq 0\right\} & \text { if } s(\overline{\mathrm{x}})<r(\overline{\mathrm{x}})=0 \\
\left\{\mathrm{~d} \in \mathbb{R}^{n}: \overline{\mathrm{y}}^{\top} \mathrm{d} \leq 0\right\} & \text { if } r(\overline{\mathrm{x}})<s(\overline{\mathrm{x}})=0 \\
\mathbb{R}^{n} & \text { if } \max \{r(\overline{\mathrm{x}}), s(\overline{\mathrm{x}})\}<0
\end{array}\right\} .
$$

This cone is sometimes called the set of linearized feasible directions NW06, Def. 12.3]. If $\bar{x}$ is locally optimal for the CDT problem (8), Slater's condition implies the local first-order condition

$$
\overline{\mathrm{g}}^{\top} \mathrm{d} \geq 0 \quad \text { for all } \mathrm{d} \in \Gamma(\overline{\mathrm{x}})
$$

which is equivalent to $\bar{x}$ being a KKT point, i.e., a feasible point satisfying the KKT conditions

$$
\overline{\mathrm{g}}+\bar{u} \overline{\mathrm{x}}+\bar{v} \overline{\mathrm{y}}=\mathrm{o} \text { and } \bar{u} r(\overline{\mathrm{x}})=\bar{v} s(\overline{\mathrm{x}})=0
$$

for some (not necessarily unique) multiplier pair $(\bar{u}, \bar{v}) \in \mathbb{R}_{+}^{2}$. We refer to $(\overline{\mathrm{x}} ; \bar{u}, \bar{v})$ as a KKT triple. Clearly, the second condition in (13) holds automatically when $\bar{x} \in B$. 


\subsection{Global optimality criteria via extremal increments}

Since $f$ is a quadratic function, it must be either convex or concave along feasible rays emanating from $\bar{x}$. If $d^{\top} Q d \geq 0$, condition 12 prohibits any feasible value along a ray given by $d \in \Gamma(\bar{x})$ from falling below the current value $f(\bar{x})$. Indeed, we have the general exact second-order expansion which will be central for the arguments in the sequel:

$$
f(\bar{x}+t d)=f(\bar{x})+t \bar{g}^{\top} \mathrm{d}+\frac{t^{2}}{2} \mathrm{~d}^{\top} \mathrm{Q} \mathrm{d} .
$$

So the only chance to improve the locally optimal value is along a feasible negative-curvature direction $\mathrm{d} \in \Gamma(\overline{\mathrm{x}})$ with $\mathrm{d}^{\top} \mathrm{Qd}<0$. For these directions, it is clear that $\bar{g}^{\top} \mathrm{d}>0$ and that the best improving value (if it exists at all) must be obtained at the extremal increment given by

$$
t_{\mathrm{d}}:=\max \{t \geq 0: \max \{r(\overline{\mathrm{x}}+t \mathrm{~d}), s(\overline{\mathrm{x}}+t \mathrm{~d})\} \leq 0\},
$$

which actually satisfies $\max \left\{r\left(\overline{\mathrm{x}}+t_{\mathrm{d}} \mathrm{d}\right), s\left(\overline{\mathrm{x}}+t_{\mathrm{d}} \mathrm{d}\right)\right\}=0$. For simplicity, assume that $\overline{\mathrm{x}} \in B$. Then, expanding $r$ and $s$ as we did for $f$ in (14) and dividing by $t_{\mathrm{d}}>0$, we obtain

$$
t_{\mathrm{d}}=\min \left\{-\frac{2 \overline{\mathrm{x}}^{\top} \mathrm{d}}{\|\mathrm{d}\|^{2}},-\frac{2 \overline{\mathrm{y}}^{\top} \mathrm{d}}{\left\|\mathrm{A}^{\top} \mathrm{d}\right\|^{2}}\right\}
$$

(observe that by strict convexity of the constraints, we only need to consider feasible directions $d \in \Gamma(\bar{x})$ where both $\bar{x}^{\top} d<0$ and $\bar{y}^{\top} d<0$ ). Thus we arrive at the following characterization of global optimality of problem (8):

Theorem 2.1 Let $\bar{x} \in B$ be a KKT point of the CDT problem (8). Then $\bar{x}$ solves (8) globally if and only if both the following homogeneous cubic conditions are satisfied:

$$
\left.\begin{array}{ll}
\left(\bar{x}^{\top} \mathrm{d}\right) \mathrm{d}^{\top} \mathrm{Qd} \leq\left(\overline{\mathrm{g}}^{\top} \mathrm{d}\right)\|\mathrm{d}\|^{2} & \text { for all } \mathrm{d} \in \Gamma^{(r)}(\overline{\mathrm{x}}) \text { and } \\
\left(\overline{\mathrm{y}}^{\top} \mathrm{d}\right) \mathrm{d}^{\top} \mathrm{Qd} \leq\left(\overline{\mathrm{g}}^{\top} \mathrm{d}\right)\left\|\mathrm{A}^{\top} \mathrm{d}\right\|^{2} & \text { for all } \mathrm{d} \in \Gamma^{(s)}(\overline{\mathrm{x}}),
\end{array}\right\}
$$

where

$$
\left.\begin{array}{l}
\Gamma^{(r)}(\bar{x}):=\left\{d \in \Gamma(\bar{x}):\left(\bar{x}^{\top} d\right)\left\|A^{\top} d\right\|^{2} \geq\left(\bar{y}^{\top} d\right)\|d\|^{2}\right\} \text { and } \\
\Gamma^{(s)}(\bar{x}):=\left\{d \in \Gamma(\bar{x}):\left(\bar{x}^{\top} d\right)\left\|A^{\top} d\right\|^{2} \leq\left(\bar{y}^{\top} d\right)\|d\|^{2}\right\} .
\end{array}\right\}
$$

Proof. First note that $\Gamma^{(r)}(\bar{x})$ is the set of all feasible directions $d$ with $t_{\mathrm{d}}=-\frac{2 \overline{\mathrm{x}}^{\top} \mathrm{d}}{\|\mathrm{d}\|^{2}}$, by 16 . Then the first inequality follows from plugging this 
$t_{\mathrm{d}}$ into the formula (14) and requiring that the extremal increment be nonnegative, i.e., $f\left(\bar{x}+t_{d} \mathrm{~d}\right)-f(\bar{x}) \geq 0$. The second condition follows in the same way.

In some sense, the above result resembles a global optimality condition for quadratic optimization problems from [Bom92] where as many conditions are needed as there are inactive constraints. However, the conditions there are homogenous quadratic, and the cones are polyhedral. Here, the quadraticity of the constraints requires us to employ cubic polynomials both for the inequalities and the cone constraints in the conditions. A similar optimality condition was presented, for the special case of concave $f$, in [HU01, Theorem 4] and derived by $\epsilon$-subdifferential calculus.

This result could easily be extended to the cases where $\bar{x} \notin B$, but this would be of little interest. Indeed, it is more concise to assume also that $\overline{\mathrm{x}}+t_{\mathrm{d}} \mathrm{d} \in B$. Then we arrive at only one cubic condition:

Theorem 2.2 Let $\bar{x} \in B$ be a KKT point of the CDT problem (8) and assume that all global solutions of (8) are contained in B. Then $\bar{x}$ solves (8) globally if and only if the following condition is satisfied:

$$
\left(\bar{x}^{\top} \mathrm{d}\right) \mathrm{d}^{\top} \mathrm{Q} \mathrm{d} \leq\left(\overline{\mathrm{g}}^{\top} \mathrm{d}\right)\|\mathrm{d}\|^{2} \text { for all } \mathrm{d} \in \Gamma^{(0)}(\overline{\mathrm{x}}),
$$

where

$$
\Gamma^{(0)}(\bar{x})=\left\{\mathrm{d} \in \mathbb{R}^{n}: \overline{\mathrm{x}}^{\top} \mathrm{d} \leq 0 \text { and }\left(\overline{\mathrm{x}}^{\top} \mathrm{d}\right)\left\|\mathrm{A}^{\top} \mathrm{d}\right\|^{2}=\left(\overline{\mathrm{y}}^{\top} \mathrm{d}\right)\|\mathrm{d}\|^{2}\right\} .
$$

Proof. For any $\mathrm{d} \in \Gamma(\overline{\mathrm{x}}) \backslash\{\mathrm{o}\}$, we have $\overline{\mathrm{x}}+t \mathrm{~d} \in B$ for some $t>0$, i.e., $r(\overline{\mathrm{x}}+t \mathrm{~d})=s(\overline{\mathrm{x}}+t \mathrm{~d})=0$, only if

$$
t=t_{d} \quad \text { and } \quad t_{d}=-\frac{2 \overline{\mathrm{x}}^{\top} \mathrm{d}}{\|\mathrm{d}\|^{2}}=-\frac{2 \overline{\mathrm{y}}^{\top} \mathrm{d}}{\left\|\mathrm{A}^{\top} \mathrm{d}\right\|^{2}},
$$

by 15 and $(16)$. So we see that $d \in \Gamma^{(r)}(\bar{x}) \cap \Gamma^{(s)}(\bar{x})=\Gamma^{(0)}(\bar{x})$ as specified in (20). By the assumptions of the theorem, we only need to check these extremal increments $f\left(\overline{\mathrm{x}}+t_{\mathrm{d}} \mathrm{d}\right)-f(\overline{\mathrm{x}}) \geq 0$ for $\mathrm{d} \in \Gamma^{(0)}(\overline{\mathrm{x}})$, where both inequality conditions in (17) coincide. Hence the result.

Notice that both inequalities in (17) and the one in (19) are automatically satisfied for directions $d \in \Gamma(\bar{x})$ with non-negative curvature $d^{\top} Q d \geq 0$ at a KKT point $\bar{x}$, by virtue of $(12)$. So, if $\bar{x}$ is a KKT point, $\Gamma(\bar{x})$-copositivity of $Q$ is a sufficient condition for global optimality. We will strengthen this result significantly in the next section. 


\subsection{Lagrangian duality}

We start with some observations on the well-studied dual problem. Most of them can be found also in [AZ09, BE06, CY99, CY09, JL13, YZ03, Yua90. The full Lagrangian function is

$$
\begin{aligned}
L(\mathrm{x} ; u, v) & =f(\mathrm{x})+u r(\mathrm{x})+v s(\mathrm{x}) \\
& =\frac{1}{2} \mathrm{x}^{\top} \mathrm{H}(u, v) \mathrm{x}+(\mathrm{q}-v \mathrm{Aa})^{\top} \mathrm{x}+\frac{1}{2}\left[v\|\mathrm{a}\|^{2}-u-v\right],
\end{aligned}
$$

where

$$
\mathrm{H}(u, v)=\mathrm{Q}+u \mathrm{I}_{n}+v \mathrm{AA}^{\top} \quad \text { for }(u, v) \in \mathbb{R}_{+}^{2}
$$

is the Hessian of $L$ w.r.t. x. Denote by

$$
\Theta(u, v)=\inf \left\{L(\mathrm{x} ; u, v): \mathrm{x} \in \mathbb{R}^{n}\right\}
$$

the Lagrangian dual function which is finite at $(u, v)$ if and only if both $\mathrm{H}(u, v)$ is psd and the equation

$$
\nabla_{\mathrm{x}} L(\mathrm{x} ; u, v)=\mathrm{H}(u, v) \mathrm{x}+\mathrm{q}-v \mathrm{Aa}=\mathrm{o}
$$

has a solution in $\mathrm{x}$.

Any KKT point $\bar{x}$ solves 23 w.r.t. at least one multiplier pair $(\bar{u}, \bar{v})$. Conversely, if there is a feasible solution $\overline{\mathrm{x}}$ to 23 for some $(\bar{u}, \bar{v}) \in \mathbb{R}_{+}^{2}$ satisfying $\bar{u} r(\overline{\mathrm{x}})+\bar{v} s(\overline{\mathrm{x}})=0$, then $\overline{\mathrm{x}}$ is a KKT point. But there are cases where, even for a KKT multiplier pair $(\bar{u}, \bar{v})$ corresponding to a global solution $\bar{x}$, the Hessian $\mathrm{H}(\bar{u}, \bar{v})$ is indefinite (recall our convention that this means it is not psd, and hence has at least one negative eigenvalue) 1$]$

The Lagrangian dual problem is

$$
z_{D}^{*}:=\sup \left\{\Theta(u, v):(u, v) \in \mathbb{R}_{+}^{n}\right\} .
$$

Note that the optimal dual variables $\left(u^{*}, v^{*}\right)$ maximizing $\Theta(u, v)$, if they exist at all, cannot coincide with a KKT multiplier pair $(\bar{u}, \bar{v})$ when $\mathbf{H}(\bar{u}, \bar{v})$ is indefinite as then $\Theta(\bar{u}, \bar{v})=-\infty$, despite the fact that

$$
\mathrm{H}(\bar{u}, \bar{v}) \overline{\mathrm{x}}+\mathrm{q}-\bar{v} \mathrm{Aa}=\overline{\mathrm{g}}+\bar{u} \mathrm{x}+\bar{v} \overline{\mathrm{y}}=\mathrm{o},
$$

i.e., that $\overline{\mathrm{x}}$ is a stationary point (but not a minimizer) of $L(. ; \bar{u}, \bar{v})$. In fact, it is shown in [NWY00] that there is a duality gap, i.e., $z_{D}^{*}<z^{*}$, if and only if, for all KKT triples $(\bar{x} ; \bar{u}, \bar{v}) \in \mathbb{R}^{n} \times \mathbb{R}_{+}^{2}$, the Hessian $\mathrm{H}(\bar{u}, \bar{v})$ is indefinite. Furthermore, it is also shown in [NWY00] that the Lagrangian dual problem coincides with the semidefinite optimization relaxation of (8). So this relaxation, too, is tight, if and only if $\mathrm{H}(\bar{u}, \bar{v})$ is psd for some multiplier pair $(\bar{u}, \bar{v}) \in \mathbb{R}_{+}^{2}$ at some KKT point $\bar{x}$, which then must be a global solution.

\footnotetext{
${ }^{1}$ JLL09, Cor.5.4] offers simple sufficient conditions on the problem data (Q, q, A, a) that rule out this phenomenon, namely that no off-diagonal entry of $Q$ or $A A^{\top}$ is strictly positive and $\{\mathrm{Aa},-\mathrm{q}\} \subseteq \mathbb{R}_{+}^{n}$. Note that this particularly implies $\mathrm{H}(\bar{u}, \bar{v}) \overline{\mathrm{x}} \in \mathbb{R}_{+}^{n}$ by $(23)$.
} 


\subsection{Copositivity on the polyhedral tangent cone}

From the previous discussion, it is quite understandable that the case of indefinite $\mathrm{H}(\bar{u}, \bar{v})$ has been considered to be difficult. We now propose a new approach to this case using copositivity, which at first sight may seem a difficult property to verify. However, while checking $\Gamma$-copositivity in the standard case where $\Gamma=\mathbb{R}_{+}^{n}$ is NP-hard [DG14, MK87], there are cones for which this condition is easy to check. The simplest example is obtained by taking any halfspace for $\Gamma$. Indeed, since $(-d)^{\top} S(-d)=d^{\top} S d$, we see that any matrix $S$ is $\Gamma$-copositive if and only if it is $\Gamma \cup-\Gamma$-copositive, so if $\Gamma$ is a halfspace, $\Gamma$-copositivity is equivalent to definiteness.

For the polyhedral tangent cone $\Gamma(\bar{x})$ defined in the previous section, the following holds.

Lemma 2.1 Let $\bar{x}$ be a feasible point of (8) and define $\Gamma(\bar{x})$ as in (11).

(a) If $\max \{r(\overline{\mathrm{x}}), s(\overline{\mathrm{x}})\}<0$, then $\Gamma(\overline{\mathrm{x}})=\mathbb{R}^{n}$;

(b) if $r(\overline{\mathrm{x}})<s(\overline{\mathrm{x}})=0$, then $\Gamma(\overline{\mathrm{x}}) \cup-\Gamma(\overline{\mathrm{x}})=\mathbb{R}^{n}$;

(c) if $s(\overline{\mathrm{x}})<r(\overline{\mathrm{x}})=0$, then $\Gamma(\overline{\mathrm{x}}) \cup-\Gamma(\overline{\mathrm{x}})=\mathbb{R}^{n}$;

(d) if $s(\overline{\mathrm{x}})=r(\overline{\mathrm{x}})=0$, then $\Gamma(\overline{\mathrm{x}})=\left\{\mathrm{d} \in \mathbb{R}^{n}: \max \left\{\overline{\mathrm{x}}^{\top} \mathrm{d}, \overline{\mathrm{y}}^{\top} \mathrm{d}\right\} \leq 0\right\}$.

Proof. Immediate from the definition (11).

Hence, the only case where $\Gamma(\bar{x})$-copositivity is different from definiteness is when $\bar{x} \in B$. We will see in Section 3 that in this case too, checking copositivity on the "wedge" $\Gamma(\bar{x})$ is not difficult.

\subsection{Global optimality conditions}

Now we can formulate a first condition which narrows the difficulty gap. Specifically, this result is stronger than the one given by [NWY00], which states that if $\mathrm{H}(\bar{u}, \bar{v})$ is psd for a $\operatorname{KKT}$ triple $(\overline{\mathrm{x}} ; \bar{u}, \bar{v}) \in \mathbb{R}^{n} \times \mathbb{R}_{+}^{2}$, then $\overline{\mathrm{x}}$ is globally optimal.

Theorem 2.3 Let $(\bar{x} ; \bar{u}, \bar{v}) \in \mathbb{R}^{n} \times \mathbb{R}_{+}^{2}$ be a KKT triple for the CDT problem (8). If $\mathrm{H}(\bar{u}, \bar{v})$ is $\Gamma(\overline{\mathrm{x}})$-copositive, then $\overline{\mathrm{x}}$ is a global solution to (8). 
Proof. Let $x \neq \bar{x}$ be (8)-feasible. Then, by convexity, $d=x-\bar{x} \in \Gamma(\bar{x})$. Further, as $L$ is a quadratic function underestimating $f$ on the primal-dual feasible set but coincides with it at $\bar{x}$, we know

$$
\begin{aligned}
f(\mathrm{x}) \geq L(\mathrm{x} ; \bar{u}, \bar{v}) & =L(\overline{\mathrm{x}} ; \bar{u}, \bar{v})+\mathrm{d}^{\top} \nabla_{\mathrm{x}} L(\overline{\mathrm{x}} ; \bar{u}, \bar{v})+\frac{1}{2} \mathrm{~d}^{\top} \mathrm{H}(\bar{u}, \bar{v}) \mathrm{d} \\
& =f(\overline{\mathrm{x}})+\frac{1}{2} \mathrm{~d}^{\top} \mathrm{H}(\bar{u}, \bar{v}) \mathbf{d} \geq f(\overline{\mathrm{x}})+0=f(\overline{\mathrm{x}}),
\end{aligned}
$$

by the copositivity assumption, using the property that $\nabla_{\mathrm{x}} L(\overline{\mathrm{x}} ; \bar{u}, \bar{v})=0$. Hence $\bar{x}$ is globally optimal.

Lemma 2.1 implies that, if $\bar{x} \notin B$, the $\Gamma(\bar{x})$-copositivity condition reduces to the familiar psd condition on $\mathrm{H}(\bar{u}, \bar{v})$, but the new result is stronger if $\bar{x} \in B$. Indeed, we shall see in Section 4 that the copositivity condition sometimes holds when the psd condition does not. Furthermore, the psd condition does not necessarily hold at a global minimizer $\bar{x}$ of the CDT problem even when just one constraint is binding: in such a case $\bar{x}$ is a local but not a global minimizer of the associated trust region problem (see Section 4).

It is well known Yua90 that at an optimal solution $\bar{x}$ of (8), the Hessian $\mathrm{H}(\bar{u}, \bar{v})$ can have at most one negative eigenvalue if the corresponding KKT multiplier pair $(\bar{u}, \bar{v})$ is unique, cf. also [PY97, Thm.3.8]. In any case, $\mathrm{H}(\bar{u}, \bar{v})$ cannot have more than two negative eigenvalues [PY97, Thm.4.3]. We now show that if the Hessian is both copositive and indefinite, the multiplier pair must be unique and the Hessian must have exactly one negative eigenvalue.

Theorem 2.4 Suppose $(\overline{\mathrm{x}} ; \bar{u}, \bar{v}) \in B \times \mathbb{R}_{+}^{2}$ is a KKT triple for the CDT problem (8). If $\mathrm{H}(\bar{u}, \bar{v})$ is $\Gamma(\overline{\mathrm{x}})$-copositive but indefinite, then

(a) the gradients $\{\bar{x}, \bar{y}\}$ of the binding constraints are linearly independent;

(b) the multipliers $(\bar{u}, \bar{v})$ are unique;

(c) the Hessian $\mathrm{H}(\bar{u}, \bar{v})$ of the Lagrangian has exactly one negative eigenvalue (counting multiplicity).

Proof. Suppose that $\overline{\mathrm{y}}=\alpha \overline{\mathrm{x}}$ for some $\alpha \in \mathbb{R}$. We know from the discussion at the end of Section 1 that $\alpha>0$. Then $\Gamma(\bar{x})=\left\{d \in \mathbb{R}^{n}: \bar{x}^{\top} d \leq 0\right\}$ is a half space, rendering $\mathrm{H}(\bar{u}, \bar{v})$ psd in contradiction of the assumption. This shows Assertion (a) which implies, by a standard linear independence constraint qualification reasoning, Assertion (b). Finally, assume there are orthogonal 
eigenvectors $\{\mathrm{b}, \mathrm{c}\}$ of $\mathrm{H}(\bar{u}, \bar{v})$ such that $(\beta \mathrm{b}+\gamma \mathrm{c})^{\top} \mathrm{H}(u, v)(\beta \mathrm{b}+\gamma \mathrm{c})<0$ for all $[\beta, \gamma]^{\top} \in \mathbb{R}^{2} \backslash\{\mathrm{o}\}$, and consider the linear system

$$
C\left[\begin{array}{l}
\beta \\
\gamma
\end{array}\right] \leq\left[\begin{array}{l}
0 \\
0
\end{array}\right] \quad \text { where } \quad C=\left[\begin{array}{ll}
b^{\top} \bar{x} & c^{\top} \bar{x} \\
b^{\top} \bar{y} & c^{\top} \bar{y}
\end{array}\right] .
$$

If $\mathrm{C}$ is singular, we can choose $[\beta, \gamma]^{\top} \in \mathbb{R}^{2} \backslash\left\{[0,0]^{\top}\right\}$ such that $C[\beta, \gamma]^{\top}=\mathrm{o}$; otherwise, choose $[\beta, \gamma]^{\top}=\mathbf{C}^{-1}[-1,-1]^{\top}$. In either case, we get $\beta \mathbf{b}+\gamma \mathbf{c} \in$ $\Gamma(\overline{\mathrm{x}})$, conflicting with the copositivity condition. So $\mathrm{H}(\bar{u}, \bar{v})$ can have at most one negative eigenvalue. On the other hand, we assumed $\mathbf{H}(\bar{u}, \bar{v})$ to be indefinite. Hence Assertion (c) follows.

We now turn to the degenerate case. We say that a KKT point $\bar{x} \in B$ is degenerate if the constraint gradients are linearly dependent, i.e., $\overline{\mathrm{y}}=\alpha \overline{\mathrm{x}}$ for some $\alpha>0$, and hence

$$
\overline{\mathrm{g}}=-(\bar{u}+\alpha \bar{v}) \overline{\mathrm{x}} .
$$

If $\overline{\mathrm{g}}=\mathrm{o}$, then the KKT multipliers $\bar{u}$ and $\bar{v}$ must both be zero. More generally,

$$
(\tilde{u}, 0)=(\|\overline{\mathrm{g}}\|, 0) \text { and }(0, \tilde{v})=\left(0, \frac{\|\overline{\mathrm{g}}\|}{\alpha}\right)
$$

are both KKT multiplier pairs for $\bar{x}$, as are all pairs in their convex hull, a line segment in $\mathbb{R}_{+}^{2}$ that we parametrize as follows:

$$
(\bar{u}(t), \bar{v}(t))=((1-t) \tilde{u}, t \tilde{v}): \quad t \in[0,1] .
$$

Because of the nonnegativity condition, no other multiplier pairs for $\bar{x}$ exist. We now show that in this degenerate case, $\bar{x}$ is globally optimal if and only if the matrix $\mathrm{H}(\bar{u}(t), \bar{v}(t))$ is psd for some $t \in[0,1]$, where

$$
\mathrm{H}(\bar{u}(t), \bar{v}(t))=\mathrm{Q}+(1-t) \tilde{u} \mathrm{I}_{n}+t \tilde{v} \mathrm{AA}^{\top}=(1-t) \mathrm{H}(\tilde{u}, 0)+t \mathrm{H}(0, \tilde{v}) .
$$

Note that if $\overline{\mathrm{g}}=\mathrm{o}$, then $\mathrm{H}(\bar{u}(t), \bar{v}(t))=\mathrm{H}(0,0)=\mathrm{Q}$ for all $t \in[0,1]$.

Theorem 2.5 Suppose $\overline{\mathrm{x}} \in B$ is a degenerate KKT point. Then $\overline{\mathrm{x}}$ is globally optimal for (8) if and only if $\mathrm{H}(\bar{u}(t), \bar{v}(t))$ is psd for some $t \in[0,1]$.

Proof. We already know NWY00 that if $\mathrm{H}(\bar{u}(t), \bar{v}(t))$ is psd for some $t$, then $\bar{x}$ is globally optimal. Now suppose $\bar{x}$ is globally optimal, and apply Theorem 2.1. Using (25) and (26), we have

$$
\overline{\mathrm{g}}=-\tilde{u} \overline{\mathrm{x}}=-\frac{\tilde{u}}{\alpha} \overline{\mathrm{y}}
$$


so (17) reduces to

$$
\left.\begin{array}{ll}
\mathrm{d}^{\top} \mathrm{Qd} \geq-\tilde{u}\|\mathrm{~d}\|^{2} & \text { for all } \mathrm{d} \text { with }\left\|\mathrm{A}^{\top} \mathrm{d}\right\|^{2} \geq \alpha\|\mathrm{d}\|^{2} \text { and } \\
\mathrm{d}^{\top} \mathrm{Qd} \geq-\tilde{u}\left\|\mathrm{~A}^{\top} \mathrm{d}\right\|^{2} / \alpha & \text { for all d with }\left\|\mathrm{A}^{\top} \mathrm{d}\right\|^{2} \leq \alpha\|\mathrm{d}\|^{2} .
\end{array}\right\}
$$

Note that we have dropped the inequality $\bar{x}^{\top} d<0$ : requiring these conditions to hold on a strict half-space is equivalent to requiring them on the whole space, since they do not depend on the sign of $d$ and the orthogonal complement of $\bar{x}$ is dense in $\mathbb{R}^{n}$. So,

$$
\mathrm{d}^{\top} \mathrm{H}(\tilde{u}, 0) \mathrm{d} \geq 0 \quad \text { for all } \mathrm{d} \text { with }\left\|\mathrm{A}^{\top} \mathrm{d}\right\|^{2} \geq \alpha\|\mathrm{d}\|^{2}
$$

and

$$
\mathrm{d}^{\top} \mathrm{H}(0, \tilde{v}) \mathrm{d} \geq 0 \quad \text { for all } \mathrm{d} \text { with }\left\|\mathrm{A}^{\top} \mathrm{d}\right\|^{2} \leq \alpha\|\mathrm{d}\|^{2} .
$$

Hence,

$$
\max \left\{\mathrm{d}^{\top} \mathbf{H}(\tilde{u}, 0) \mathrm{d}, \mathrm{d}^{\top} \mathrm{H}(0, \tilde{v}) \mathrm{d}\right\} \geq 0 \quad \text { for all } \mathrm{d} \in \mathbb{R}^{n} .
$$

Now we apply a result on matrix pencils due to Yuan YYua90, Lemma 2.3] showing that there must therefore exist $t \in[0,1]$ such that $\mathbf{H}(\bar{u}(t), \bar{v}(t))$ given in $(28)$ is psd.

\subsection{Local optimality conditions}

We continue by discussing a well-known set of conditions for local optimality. To this end, we introduce the reduced polyhedral tangent cone comprising all feasible directions along which no first-order change in the objective is possible:

$$
\Gamma_{\text {red }}(\bar{x}):=\left\{d \in \Gamma(\bar{x}): \bar{g}^{\top} d=0\right\} .
$$

We first present results on $\Gamma_{\text {red }}(\bar{x})$ that are similar to those given in Lemma 2.1 for $\Gamma(\bar{x})$, but now specifically for KKT points $\bar{x}$, and with a focus on the signs of the multipliers $\bar{u}, \bar{v}$.

Lemma 2.2 Let $(\bar{x} ; \bar{u}, \bar{v}) \in \mathbb{R}^{n} \times \mathbb{R}_{+}^{2}$ be a KKT triple for (8). Then:
(a) If $\bar{u}=\bar{v}=0$, then $\Gamma_{\text {red }}(\overline{\mathrm{x}})=\Gamma(\overline{\mathrm{x}}) \supset\{\overline{\mathrm{x}}, \overline{\mathrm{y}}\}^{\perp}$;
(b) if $\bar{u}=0<\bar{v}$, then $\Gamma_{\text {red }}(\overline{\mathrm{x}})=\overline{\mathrm{y}}^{\perp}$;
(c) if $\bar{v}=0<\bar{u}$, then $\Gamma_{\text {red }}(\overline{\mathrm{x}})=\overline{\mathrm{x}}^{\perp}$;
(d) if $\min \{\bar{u}, \bar{v}\}>0$, then $\Gamma_{\text {red }}(\overline{\mathrm{x}})=\{\overline{\mathrm{x}}, \overline{\mathrm{y}}\}^{\perp}$. 
Proof. The cases now position $\bar{g}$ in relation to $\{\bar{x}, \bar{y}\}$, since $\bar{g}=-u \bar{x}-v \bar{y}$. The claim follows from the definitions $(33)$ and (11).

Using $\Gamma_{\text {red }}(\bar{x})$-copositivity conditions, in a more or less explicit way, for second-order necessary conditions is by now fairly standard even for more general smooth optimization problems; see, e.g. Bor82, Con80, Maj71, or for a recent and slightly more general treatment, Bom13. However, in the degenerate case (25) the KKT multipliers are not unique (unless $\overline{\mathrm{g}}=\mathrm{o}$, which means (a) above applies). In such cases constraint qualifications that are needed by the standard theory do not hold for our problem and we need a more careful analysis. When the multipliers are not unique, all of (b), (c) and (d) above apply for different choices of the multiplier pair $(\bar{u}, \bar{v})$, but this is not a contradiction as all three equations reduce to

$$
\Gamma_{\text {red }}(\bar{x})=\bar{x}^{\perp}=\bar{y}^{\perp}=\bar{g}^{\perp} .
$$

Assume that $\overline{\mathrm{x}}$ is a degenerate KKT point and that $\overline{\mathrm{g}} \neq 0$, let $t \in[0,1]$ be given, defining the KKT multiplier pair $(\bar{u}(t), \bar{v}(t))$ associated with $\overline{\mathrm{x}}$ as in (27), and define the reduced feasible set

$$
M_{t}:=\left\{\mathrm{x} \in \mathbb{R}^{n}: r(\mathrm{x}) \leq 0, s(\mathrm{x}) \leq 0 \text { and } \bar{u}(t) r(\mathrm{x})+\bar{v}(t) s(\mathrm{x})=0\right\}
$$

which is strictly contained in the feasible set

$$
M=\left\{\mathrm{x} \in \mathbb{R}^{n}: r(\mathrm{x}) \leq 0 \text { and } s(\mathrm{x}) \leq 0\right\} .
$$

The reduced polyhedral cone $\Gamma_{\text {red }}(\bar{x})$ plays essentially the same role for $M_{t}$ as $\Gamma(\overline{\mathrm{x}})$ does for $M$. The key difference is that $M$ is convex with non-empty interior by assumption (4) whereas $M_{t}$ is non-convex and has no interior points. Furthermore, even the weakest of the constraint qualifications employed in the recent paper Bom13] (specifically, the reflected Abadie constraint qualification) need not be satisfied at $\bar{x}$ for the reduced feasible set $M_{t}$.

Before we give a general analysis, let us look at the planar case $n=$ 2 for illustrative purposes; we will also need this for arguments proving Theorem 2.6 below which investigate the planar section $M \cap \operatorname{span}\{\overline{\mathrm{x}}, \mathrm{d}\}$ for arbitrary $\mathrm{d} \in \Gamma_{\text {red }}(\overline{\mathrm{x}})$. If $n=2$, then the boundary of the first CDT constraint, $C=\{\mathrm{x}: r(\mathrm{x})=0\}$, is a circle and that of the second constraint, $E=\{\mathrm{x}: s(\mathrm{x})=0\}$, is an ellipse, and by definition, we have $M_{0}=C \cap M$ and $M_{1}=E \cap M$. Since $\bar{y}=\alpha \bar{x}$ for $\alpha>0, C$ and $E$ are tangent to each other at $\bar{x}$ but they may have different curvatures there. If the curvature of $C$ is smaller than that of $E$ locally around $\bar{x}$, then locally, $E$ is contained in $M$ 
so $M_{1}$ and $E$ coincide locally, but $M_{0}$ contains only the point $\bar{x}$. To be more precise, there is an $\varepsilon>0$ such that, for the disc $D_{\varepsilon}=\{\mathrm{x}:\|\mathrm{x}-\overline{\mathrm{x}}\|<\varepsilon\}$, we have

$$
M_{1} \cap D_{\varepsilon}=E \cap M \cap D_{\varepsilon}=E \cap D_{\varepsilon} \quad \text { but } \quad M_{0} \cap D_{\varepsilon}=C \cap M \cap D_{\varepsilon}=\{\bar{x}\} .
$$

Similarly, if the curvature of $C$ is larger than that of $E$ locally around $\bar{x}$, then

$$
M_{0} \cap D_{\varepsilon}=C \cap M \cap D_{\varepsilon}=C \cap D_{\varepsilon} \quad \text { but } \quad M_{1} \cap D_{\varepsilon}=E \cap M \cap D_{\varepsilon}=\{\bar{x}\} .
$$

So, when the curvatures are not equal, any vector $d \in \Gamma_{\text {red }}(\bar{x})=x^{\perp}$ (actually, it is unique up to a scalar multiple) is tangent at $\bar{x}$ to a feasible trajectory in either $M_{0}$ or $M_{1}$.

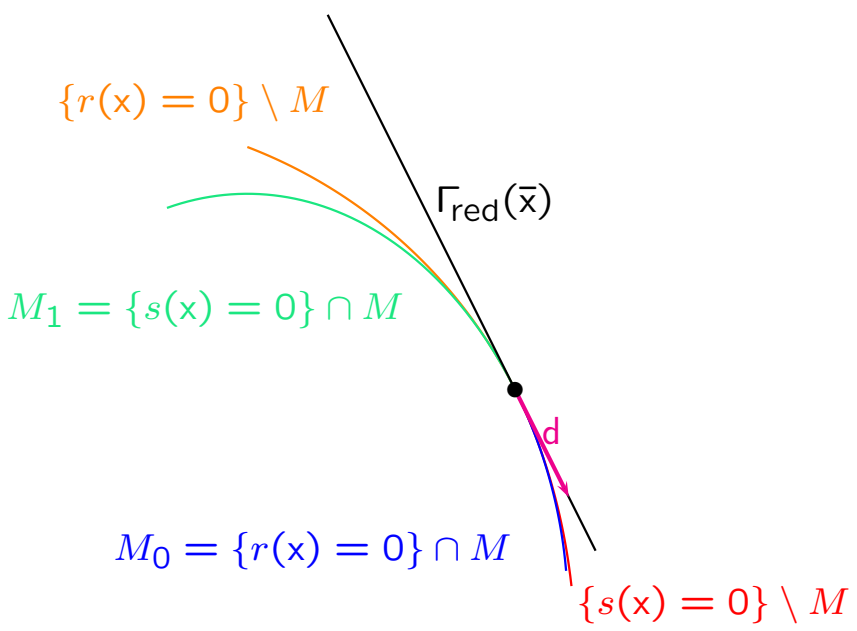

Figure 1: Osculation of $C$ (orange/blue) and $E$ (red/green) at a non-apex $\bar{x}$

But what happens if both curvatures coincide, i.e., if $C$ is the circle osculating $E$ at $\bar{x}$ ? Only if $\bar{x}$ is one of the apexes of $E$, do we have either of the situations described above. Otherwise, the situation is as depicted in Figure 1, with

$M_{0} \cap D_{\varepsilon}=C \cap M \cap D_{\varepsilon}=C^{+} \cap D_{\varepsilon} \quad$ and $\quad M_{1} \cap D_{\varepsilon}=E \cap M \cap D_{\varepsilon}=E^{-} \cap D_{\varepsilon}$,

where $C^{+}$is a half-circle and $D^{-}$is a half-ellipse, both emanating from $\bar{x}$ but in opposite directions. Thus, for $d \in \Gamma_{\text {red }}(\bar{x})$, either $d$ is tangent to a 
trajectory in $M_{0}$ at $\overline{\mathrm{x}}$ and and $-\mathrm{d}$ is tangent to a trajectory in $M_{1}$ at $\overline{\mathrm{x}}$, or vice versa.

So, in the degenerate case with $n=2$ we have three different cases: $E$ is locally inside $C, C$ is locally inside $E$, or neither (the case depicted in Figure 1). But in all three cases, for either $t=0$ or $t=1$, it is the case that for every vector $d \in \Gamma_{\text {red }}(\bar{x})$, either $d$ or $-d$ is a starting tangent vector for a feasible trajectory $\{\mathrm{x}(\tau): \tau \geq 0\}$ contained in $M_{t}$. Hence

$$
\begin{aligned}
f(\mathrm{x}(\tau)) & =L(\mathrm{x}(\tau) ; \bar{u}(t), \bar{v}(t)) \\
& =L(\overline{\mathrm{x}} ; \bar{u}(t), \bar{v}(t))+\frac{\tau^{2}}{2} \mathrm{~d}^{\top} \mathbf{H}(\bar{u}(t), \bar{v}(t)) \mathrm{d}+\mathfrak{o}\left(\tau^{2}\right),
\end{aligned}
$$

implying that if $\bar{x}$ is locally optimal, then $\mathrm{d}^{\top} \mathrm{H}(\bar{u}(t), \bar{v}(t)) \mathrm{d} \geq 0$. In summary, in the degenerate case with $n=2$, a necessary condition for $\bar{x}$ to be locally optimal is that for either $t=0$ or $t=1, \mathrm{H}(\bar{u}(t), \bar{v}(t))$ is $\Gamma_{\text {red }}(\overline{\mathrm{x}})$-copositive.

However, the case $n>2$ is different, requiring a more refined reasoning which exploits the special structure of $\Gamma_{\text {red }}(\bar{x})$ as expressed in Lemma 2.2. To this end, we need an extension of Yuan's lemma that we used in Section 2.5 . this is given by [JLL09, Thm.3.3]. See also [PT07] for a thorough survey on the S-Lemma which is closely related to these results.

Lemma 2.3 Let $\mathrm{C}$ and $\mathrm{D}$ be two symmetric $n \times n$ matrices and $\Gamma$ a cone such that $\Gamma \cup-\Gamma$ is a linear subspace of $\mathbb{R}^{n}$. If

$$
\max \left\{\mathrm{x}^{\top} \mathrm{Cx}, \mathrm{x}^{\top} \mathrm{Dx}\right\} \geq 0 \quad \text { for all } \mathrm{x} \in \Gamma,
$$

then there is a $t \in[0,1]$ such that $\mathrm{S}_{t}:=t \mathrm{C}+(1-t) \mathrm{D}$ is $\Gamma$-copositive.

Proof. The assertion is a paraphrase of [JLL09, Thm.3.3].

Remark 2.1 We are not aware of any extension of Lemma 2.3 to cones $\Gamma$, if $\Gamma \cup-\Gamma$ is not a linear subspace, even if $\Gamma$ were a polyhedral cone. This is the reason for emphasizing the structure of $\Gamma_{\text {red }}(\bar{x})$ in Lemma 2.2.

Now we are ready to prove our main result on local optimality conditions.

Theorem 2.6 Let $\bar{x} \in B$ be a KKT point for (8).

(a) If $\mathrm{H}(\bar{u}, \bar{v})$ is strictly $\Gamma_{\text {red }}(\overline{\mathrm{x}})$-copositive for some multiplier pair $(\bar{u}, \bar{v}) \in$ $\mathbb{R}_{+}^{2}$ satisfying the KKT conditions (13), then $\overline{\mathrm{x}}$ is a local minimizer of (8); 
(b) If $\overline{\mathrm{x}}$ is a local minimizer of (8) then $\mathrm{H}(\bar{u}, \bar{v})$ is $\Gamma_{\text {red }}(\overline{\mathrm{x}})$-copositive for some multiplier pair $(\bar{u}, \bar{v}) \in \mathbb{R}_{+}^{2}$ satisfying the KKT conditions (13).

Proof. (a) is standard and can be found, e.g., in [Bor82, Bom13]. In the nondegenerate case, meaning that either $\bar{x} \notin B$ or $\bar{x} \in B$ with $\bar{x}$ and $\bar{y}$ linearly independent, (b) is also standard: see e.g. [Bom13, Thm.1.1]. When $\mathrm{x}$ is degenerate and $\overline{\mathrm{g}}=\mathrm{o}$, the only possible KKT multiplier pair $(\bar{u}, \bar{v})$ is $(0,0)$, so again standard results apply to establish $(\mathrm{b})$. Indeed, $\Gamma_{\text {red }}(\bar{x})=$ $\Gamma(\overline{\mathrm{x}})$, cf. Lemma $2.2(\mathrm{a})$, and the counterpart of $M_{0}=M$ satisfy Abadie's constraint qualification even if $\{\bar{x}, \bar{y}\}$ are linearly dependent, so Bom13, Thm.1.1] applies, too. So it remains to deal with the degenerate case (25) with $\bar{g} \neq 0$. For any given $d \in \Gamma_{\text {red }}(\bar{x})$, we may restrict our analysis to the planar section $M \cap \operatorname{span}\{\overline{\mathrm{x}}, \mathrm{d}\}$ and conclude that either $\mathrm{d}$ or $-\mathrm{d}$ is a starting tangent vector for a feasible trajectory $\{x(\tau): \tau \geq 0\}$ contained in $M_{t} \cap \operatorname{span}\{\overline{\mathrm{x}}, \mathrm{d}\}$ where either $t=0$ or $t=1$. Hence, using (35), we conclude that since $\overline{\mathrm{x}}$ is locally optimal, it must be the case that $\mathbf{d}^{\top} \mathbf{H}(\bar{u}(t), \bar{v}(t)) \mathrm{d} \geq 0$ for either $t=0$ or $t=12$ So, we have

$$
\max \left\{\mathrm{d}^{\top} \mathrm{H}(\tilde{u}, 0) \mathrm{d}, \mathrm{d}^{\top} \mathrm{H}(0, \tilde{v}) \mathrm{d}\right\} \geq 0 \quad \text { for all } \mathrm{d} \in \Gamma_{\text {red }}(\overline{\mathrm{x}}) .
$$

Next recall from (34) that in the degenerate case, $\Gamma_{\text {red }}(\bar{x})$ is a linear subspace of $\mathbb{R}^{n}$ with codimension one. Hence, applying Lemma 2.3 , we conclude that there exists $t \in[0,1]$ such that $\mathrm{H}(\bar{u}(t), \bar{v}(t))$ given in $(28)$ is $\Gamma_{\text {red }}(\bar{x})$-copositive, in other words, there is a $t$ such that the condition $\mathrm{d}^{\top} \mathrm{H}(\bar{u}(t), \bar{v}(t)) \mathrm{d} \geq 0$ holds for all $d \in \Gamma_{\text {red }}(\bar{x})$.

Remark 2.2 Consider again Lemma 2.2. In case (a), $\Gamma_{\text {red }}(\bar{x})=\Gamma(\bar{x})$, so according to Theorem 2.4. $\Gamma_{\text {red }}(\overline{\mathrm{x}})$-copositivity of $\mathrm{H}(\bar{u}, \bar{v})$ implies that $\mathrm{H}(\bar{u}, \bar{v})$ has at most one negative eigenvalue in this case. In cases (b), (c) and (d) of Lemma 2.2. $\Gamma_{\text {red }}(\bar{x})$ is a linear subspace of codimension one or two, so $\Gamma_{\text {red }}(\overline{\mathrm{x}})$-copositivity of $\mathrm{H}(\bar{u}, \bar{v})$ immediately implies that the number of negative eigenvalues of $\mathrm{H}(\bar{u}, \bar{v})$ is at most two, with the number equal to two only if $\overline{\mathrm{x}}$ is a nondegenerate KKT point with both constraints binding and both multipliers nonzero.

\footnotetext{
${ }^{2}$ We are indebted to W. Schachinger for this observation (personal communication). Note that when $n=2$, this means that $\mathrm{H}(\bar{u}(t), \bar{v}(t))$ is $\Gamma_{\text {red }}(\overline{\mathrm{x}})$-copositive for either $t=0$ or $t=1$, but in higher dimensions the relations between the curvatures vary with $\mathrm{d}$, so that whether $t$ is 0 or 1 in the condition $\mathbf{d}^{\top} \mathbf{H}(\bar{u}(t), \bar{v}(t)) \mathbf{d} \geq 0$ depends on $\mathbf{d}$.
} 


\subsection{Summary: a hierarchy of optimality conditions}

It is convenient here to use $\psi(\mathrm{M})$ to denote the number of negative eigenvalues of a symmetric matrix $\mathrm{M}$, counting their multiplicities. Let $(\overline{\mathrm{x}} ; \bar{u}, \bar{v})$ be a nondegenerate KKT triple for (8). Then the following implications hold:

$$
\begin{array}{ll} 
& \mathrm{H}(\bar{u}, \bar{v}) \text { is positive semidefinite } \\
\Rightarrow \quad & \mathrm{H}(\bar{u}, \bar{v}) \text { is } \Gamma(\overline{\mathrm{x}}) \text {-copositive } \\
\Rightarrow \quad & \overline{\mathrm{x}} \text { solves CDT globally and } \psi(\mathrm{H}(\bar{u}, \bar{v})) \leq 1 ; \\
& \\
& \mathrm{H}(\bar{u}, \bar{v}) \text { is strictly } \Gamma_{\text {red }}(\overline{\mathrm{x}}) \text {-copositive } \\
\Rightarrow \quad & \overline{\mathrm{x}} \text { solves CDT locally } \\
\Rightarrow \quad & \mathrm{H}(\bar{u}, \bar{v}) \text { is } \Gamma_{\text {red }}(\overline{\mathrm{x}}) \text {-copositive } \\
\Rightarrow \quad & \psi(\mathrm{H}(\bar{u}, \bar{v})) \leq 2 .
\end{array}
$$

Stronger results hold in the degenerate case. Let $\bar{x}$ be a degenerate KKT point for (8), with the line segment of multiplier pairs in $\mathbb{R}_{+}^{2}$ given in (27). Then the following equivalence and implications hold:

$$
\begin{array}{ll} 
& \mathrm{H}(\bar{u}(t), \bar{v}(t)) \text { is positive semidefinite for some } t \in[0,1] \\
\Leftrightarrow \quad & \overline{\mathrm{x}} \text { solves CDT globally; } \\
& \mathrm{H}(\bar{u}(t), \bar{v}(t)) \text { is strictly } \Gamma_{\text {red }}(\overline{\mathrm{x}}) \text {-copositive for some } t \in[0,1] \\
\Rightarrow \quad & \overline{\mathrm{x}} \text { solves CDT locally } \\
\Rightarrow \quad & \mathrm{H}(\bar{u}(t), \bar{v}(t)) \text { is } \Gamma_{\text {red }}(\overline{\mathrm{x}}) \text {-copositive for some } t \in[0,1] \\
\Rightarrow \quad & \psi(\mathrm{H}(\bar{u}(t), \bar{v}(t))) \leq 1 \text { for some } t \in[0,1] .
\end{array}
$$

\section{$3 \quad$ Verifying the copositivity conditions}

In cases (b), (c) and (d) of Lemma 2.2, the reduced polyhedral tangent cone $\Gamma_{\text {red }}(\bar{x})$ is a linear subspace, say $S$, with codimension one or two. Let $Z$ be a matrix whose columns form an orthonormal basis for $S$. Then $S$ copositivity of $B$ is equivalent to positive semidefiniteness of $Z^{\top} B Z$ which can be checked by computing its Cholesky factorization using pivoting Hig02. In the case of verifying strict copositivity, pivoting is not required: if the Cholesky factorization of $Z^{\top} B Z$ using exact arithmetic breaks down without pivoting, $Z^{\top} B Z$ is not positive definite. In case (a) of Lemma 2.2, $\Gamma_{\text {red }}(\bar{x})$ equals $\Gamma(\bar{x})$. 
Lemma 2.1 characterizes the polyhedral tangent cone $\Gamma(\bar{x})$. We have already mentioned that copositivity on a half-space is equivalent to positive semidefiniteness on the whole space. So, the only challenging case is when $\bar{x} \in B$, in which case $\Gamma(\bar{x})$ is the wedge $\left\{d \in \mathbb{R}^{n}: \max \left\{\bar{x}^{\top} d, \bar{y}^{\top} d\right\} \leq 0\right\}$. First, we characterize copositivity for the canonical quarter-space in $\mathbb{R}^{n}$.

Theorem 3.1 Let $\Upsilon=\mathbb{R}_{+}^{2} \times \mathbb{R}^{n-2}$ and partition an $n \times n$ matrix $\mathrm{B}$ as follows:

$$
\mathrm{B}=\left[\begin{array}{cc}
\mathrm{R} & \mathrm{S}^{\top} \\
\mathrm{S} & \mathrm{M}
\end{array}\right] \text { where } \mathrm{R} \text { is a } 2 \times 2 \text {-matrix. }
$$

Then $\mathrm{B}$ is $\Upsilon$-copositive if and only if the following two conditions hold:

(a) $\mathrm{M}$ is positive semidefinite and $\mathrm{MM}^{\dagger} \mathrm{S}=\mathrm{S}$, i.e., ker $\mathrm{M} \subseteq$ ker $\mathrm{S}$;

(b) $\mathrm{R}-\mathrm{S}^{\top} \mathrm{M}^{\dagger} \mathrm{S}$ is positive semidefinite or has no negative entries.

Here $\mathrm{M}^{\dagger}$ is the Moore-Penrose pseudoinverse of $\mathrm{M}$.

Proof. Suppose B is $\Upsilon$-copositive. Let $y \in \mathbb{R}_{+}^{2}$ and $z \in \mathbb{R}^{n-2}$ constitute an arbitrary vector $\mathrm{x}=\left[\mathrm{y}^{\top}, \mathrm{z}^{\top}\right]^{\top} \in \Upsilon$ and define

$$
h_{\mathrm{y}}(\mathrm{z})=\mathrm{x}^{\top} \mathrm{Bx}=\mathrm{y}^{\top} \mathrm{Ry}+2 \mathrm{z}^{\top} \mathrm{Sy}+\mathrm{z}^{\top} \mathrm{Mz} .
$$

Fixing $\mathrm{y}=\mathrm{o}$ gives $0 \leq h_{\mathrm{o}}(\mathrm{z})=\mathrm{z}^{\top} \mathrm{Mz}$ for all $\mathrm{z} \in \mathbb{R}^{n-2}$ and therefore definiteness of M. Now, given any $y \in \mathbb{R}_{+}^{2}$, the function $h_{\mathrm{y}}$ must be bounded from below and therefore attains its minimum at $z_{y}=-M^{\dagger} S y$. Further, since $\mathrm{Mz}_{\mathrm{y}}=-$ Sy for all $\mathrm{y} \in \mathbb{R}_{+}^{2}$, we also have $\mathrm{MM}^{\dagger}$ Sy $=$ Sy for all $\mathrm{y} \in \mathbb{R}^{2}$, so $\mathrm{MM}^{\dagger} \mathrm{S}=\mathrm{S}$. Finally, the condition

$$
0 \leq h_{\mathrm{y}}\left(\mathrm{z}_{\mathrm{y}}\right)=\mathrm{y}^{\top}\left(\mathrm{R}-\mathrm{S}^{\top} \mathrm{M}^{\dagger} \mathrm{S}\right) \mathrm{y} \quad \text { for all } \mathrm{y} \in \mathbb{R}_{+}^{2}
$$

establishes $\mathbb{R}_{+}^{2}$-copositivity of $\mathrm{R}-\mathrm{S}^{\top} \mathrm{M}^{\dagger} \mathrm{S}$. But it is well known (and easy to see) that an $\mathbb{R}_{+}^{2}$-copositive matrix of order two with a negative entry must be positive semidefinite. Sufficiency follows by the reverse argument noting $\mathrm{x}^{\top} \mathrm{Bx}=h_{\mathrm{y}}(\mathrm{z}) \geq h_{\mathrm{y}}\left(\mathrm{z}_{\mathrm{y}}\right)$ if $\mathrm{M}$ is psd with $\mathrm{MM}^{\dagger} \mathrm{S}=\mathrm{S}$.

Finally we need to relate $\Gamma(\bar{x})$-copositivity to $\Upsilon$-copositivity. Choose any orthonormal basis $\left\{\mathrm{z}_{3}, \ldots, \mathrm{z}_{n}\right\}$ of $\{\overline{\mathrm{x}}, \overline{\mathrm{y}}\}^{\perp}$ and complete this by choosing two unit norm vectors $\mathbf{z}_{i}, i=1,2$, satisfying $\mathbf{z}_{1}^{\top} \overline{\mathrm{x}}<0=\mathbf{z}_{1}^{\top} \overline{\mathrm{y}}$ and $\mathrm{z}_{2}^{\top} \overline{\mathrm{y}}<0=\mathrm{z}_{2}^{\top} \overline{\mathrm{x}}$. Then set $Z=\left[z_{1}, \ldots, z_{n}\right]$, so

$$
\mathbf{Z}^{\top} \mathbf{Z}=\left[\begin{array}{ll}
\mathbf{U} & O^{\top} \\
O & \mathrm{I}_{n-2}
\end{array}\right] \quad \text { where } \quad \mathbf{U}=\left[\begin{array}{cc}
1 & \rho \\
\rho & 1
\end{array}\right] \quad \text { with } \rho=\mathbf{z}_{1}^{\top} \mathbf{z}_{2}
$$


Clearly, $\mathrm{H}$ is $\Gamma(\overline{\mathrm{x}})$-copositive if and only if $\mathrm{B}=\mathrm{Z}^{\top} \mathrm{HZ}$ is $\Upsilon$-copositive.

Checking $\mathbb{R}_{+}^{n}$-copositivity of a matrix $\mathrm{B}$ is hard in general although it is known that it can be done in polynomial time if $\psi(\mathrm{B}) \leq 2$ or $\psi(\mathrm{B})=$ $n-1$ [Din96, Jar13]. Here we make no assumption on the inertia of $\mathrm{B}$, but we need instead only to check copositivity on the canonical quarter space $\Upsilon \supset \mathbb{R}_{+}^{n}$. As is clear from Theorem 3.1, this can be done efficiently.

\section{Numerical experiments}

We conducted some numerical experiments to observe how often the various cases occurred on randomly generated CDT problems. We obtained feasible problems with $m=n$ as follows: the entries of $\mathrm{Q}, \mathrm{A}, \mathrm{q}$ and a were independently generated from the normal distribution, and $Q$ was replaced by its real symmetric part; then a vector $\tilde{x}$ was generated in the same way, normalized to have length one, and then $A$ and a were scaled by $1 /\left\|A^{\top} \tilde{x}-a\right\|$, guaranteeing the existence of at least one feasible point and therefore, generically, that the Slater condition holds. The vector $\tilde{x}$ was then discarded. A candidate $\bar{x}$ for the global solution of each problem was then obtained by a local optimization method $]^{3}$

We then tested the psd condition (37), the $\Gamma(\bar{x})$-copositivity condition (38) and the $\Gamma_{\text {red }}(\bar{x})$-copositivity condition (39) listed in the sequence of implications in Section 2.7 for each of 10,000 randomly generated problems for each dimension $n$ from 42 to 8 .

Specifically, the second, third and fourth columns of Table 1 report the number of times that each of (37), (38) but not (37), and (39) but not (38) occurred for the instances where two constraints are binding at $\bar{x}$. Since conditions (37) and (38) are mathematically equivalent when just one constraint is binding, it is only necessary to count the cases that (37), and (39) but not (37), hold, while all three conditions (37), (38) and (39) are

\footnotetext{
${ }^{3}$ We used BFGS to minimize the exact penalty function $p(\mathrm{x})=f(\mathrm{x})+\rho \max (r(\mathrm{x}), 0)+$ $\rho \max (s(\mathrm{x}), 0)$, for some $\rho>0$ that is increased as needed to ensure feasibility. As discussed in [L13, BFGS is a surprisingly effective method to find local minimizers of nonsmooth, nonconvex functions such as $p$. Since one can expect only to find local minimizers in general, we did this repeatedly from 10 randomly generated starting points for each problem instance, selecting the result $\bar{x}$ with the lowest value of $f$ as our candidate for the global minimizer. The results summarized in Table 1 and discussed below show that in by far the majority of cases, global optimality was confirmed, and in all except one of 70,000 tests at least local optimality was confirmed. (At the suggestion of M. Fampa, we also conducted a similar experiment using up to $2^{n}$ starting points instead of 10 , stopping as soon as global optimality was confirmed; the outcome was similar.)

${ }^{4}$ The case $n=2$ receives particular attention in the recent theoretical study YB13.
} 
equivalent when no constraint is binding so we need only check whether (37) holds. Thus, there are a total of six different cases counted in each line of Table 1. No other cases can occur in exact arithmetic, assuming that all computed solutions $\bar{x}$ are at least locally optimal, and remarkably, even in inexact arithmetic, in only one case out of 70,000 was local optimality not confirmed. Note also that although cases (39) and (40) are mathematically distinct, they are not distinguishable using inexact arithmetic, and so we may regard them as numerically equivalent. Since the problems were randomly generated, we need not be concerned about the degenerate case or cases where strict complementarity does not hold.

We see from Table 1 that when two constraints are binding at $\bar{x}$, by far the most common scenario is that $\mathrm{H}(\bar{u}, \bar{v})$ is psd, but the case that it is $\Gamma(\overline{\mathrm{x}})-$ copositive but not psd occurs with a nonzero probability. Not surprisingly, this probability decreases as $n$ increases. The second most likely scenario with two binding constraints is that neither condition holds, indicating that there is still scope for further theoretical work to close the CDT "difficulty gap" in characterizing global minima, but as long as the $\Gamma_{\text {red }}(\bar{x})$-copositivity condition holds strictly, we are assured that $\bar{x}$ is at least a local minimizer.

When only one constraint is binding, although the psd condition on $\mathrm{H}(\bar{u}, \bar{v})$ (equivalently, $\Gamma(\overline{\mathrm{x}})$-copositivity) usually holds, this is not always the case. This might seem surprising since $\mathbf{H}(\bar{u}, \bar{v})$ is always psd at the global solution of a trust-region problem, but the explanation is simple: a global solution to the CDT problem with only one binding constraint must always be a local solution to the corresponding trust-region problem, but the global solution of the trust-region problem may be infeasible for the CDT problem. In these cases, as long as the $\Gamma_{\text {red }}(\bar{x})$-copositivity condition holds strictly, we are assured that the computed solution is at least a local minimizer of the CDT problem.

When no constraint is binding, all three conditions are equivalent and guarantee that $\bar{x}$ is a global minimizer. However, the probability that this case occurs decreases rapidly as $n$ increases, because a necessary condition is that the randomly generated matrix $Q$ is psd.

Acknowledgements. The first author is grateful to Cambridge University and to New York University for the hospitality he enjoyed during his research stays as a visiting fellow. The second author was supported in part by the U.S. National Science Foundation under grant DMS-1317205. Both authors are indebted to an anonymous referee and the Managing Guest Editor for their diligence and helpful constructive remarks. 


\begin{tabular}{|c|c|c|c|c|c|c|}
\hline \# binding & 2 & 2 & 2 & 1 & 1 & 0 \\
\hline condition & psd & $\Gamma$-copos & $\Gamma_{\text {red-copos }}$ & psd & $\Gamma_{\text {red-copos }}$ & psd \\
\hline$n=2$ & 2591 & 56 & 215 & 6455 & 488 & 194 \\
$n=3$ & 3618 & 50 & 448 & 5572 & 296 & 16 \\
$n=4$ & 4214 & 39 & 418 & 5151 & 178 & 0 \\
$n=5$ & 4396 & 40 & 409 & 5043 & 112 & 0 \\
$n=6$ & 4582 & 26 & 361 & 4954 & 77 & 0 \\
$n=7$ & 4646 & 18 & 291 & 4985 & 60 & 0 \\
$n=8$ & 4688 & 14 & 244 & 5007 & 47 & 0 \\
\hline
\end{tabular}

Table 1: Number of times the psd and copositivity conditions on $\mathbf{H}(\bar{u}, \bar{v})$ occur at computed minimizers $\bar{x}$ of 10,000 randomly generated instances of feasible CDT problems for each $n$ from 2 to 8 , categorized by the number of binding constraints at $\bar{x}$.

\section{References}

[AZ09] Wenbo Ai and Shuzhong Zhang. Strong duality for the CDT subproblem: a necessary and sufficient condition. SIAM J. Optim., 19(4):1735-1756, 2009.

[BA13] Samuel Burer and Kurt Anstreicher. Second-order-cone constraints for extended trust-region subproblems. SIAM J. Optim., 23(1):432-451, 2013.

[BE06] Amir Beck and Yonina Eldar. Strong duality in nonconvex quadratic optimization with two quadratic constraints. SIAM J. Optim., 17(3):844860, 2006.

[BM14] Daniel Bienstock and Alexander Michalka. Polynomial solvability of variants of the trust-region subproblem. In Proceedings of the Twenty-Fifth Annual ACM-SIAM Symposium on Discrete Algorithms, pages 380-390, Portland, Oregon, USA, January 2014.

[Bom92] Immanuel M. Bomze. Copositivity conditions for global optimality in indefinite quadratic programming problems. Czechosl. J. Operations Res., $1: 7-19,1992$.

[Bom13] Immanuel M. Bomze. Copositivity for second-order optimality conditions in general smooth optimization problems. Preprint NI13033-POP, Isaac Newton Institute, Cambridge UK, 2013.

[Bor82] Jonathan M. Borwein. Necessary and sufficient conditions for quadratic minimality. Numer. Funct. Anal. Optim., 5:127-140, 1982.

[CDT85] Maria Rosa Celis, John E. Dennis Jr., and Richard A. Tapia. A trust region strategy for nonlinear equality constrained optimization. In $\mathrm{Nu}$ merical Optimization, 1984 (Boulder, Colo., 1984), pages 71-82. SIAM, Philadelphia, PA, 1985. 
[CGT00] Andrew R. Conn, Nicholas I. M. Gould, and Philippe L. Toint. Trustregion Methods. MPS/SIAM Series on Optimization. Society for Industrial and Applied Mathematics (SIAM), Philadelphia, PA, 2000.

[Con80] Luis B. Contesse. Une caractérisation complète des minima locaux. $\mathrm{Nu}$ mer. Math., 34:315-332, 1980.

[CY99] Xiong-Da Chen and Ya-Xiang Yuan. On local solutions of the CelisDennis-Tapia subproblem. SIAM J. Optim., 10(2):359-383, 1999.

[CY09] Xiong-Da Chen and Ya-Xiang Yuan. Strong duality for the CDT subproblem: a necessary and sufficient condition. J. Comp. Math., 19(2):113-124, 2009.

[DG14] Peter J. C. Dickinson and Luuk Gijben. On the computational complexity of membership problems for the completely positive cone and its dual. Comput. Optim. Appl., 57(2):403-415, 2014.

[Din96] Baoyan Ding. A Parametric Solution for Local and Global Optimization. PhD thesis, University of Waterloo, Ontario, Canada, 1996.

[GP05] Dima Grigoriev and Dmitrii V. Pasechnik. Polynomial time computing over quadratic maps I. Sampling in real algebraic sets. Comput. Complex., 14:20-52, 2005.

[Hig02] Nicholas J. Higham. Accuracy and Stability of Numerical Algorithms. Society for Industrial and Applied Mathematics (SIAM), Philadelphia, PA, second edition, 2002.

[HU01] Jean-Baptiste Hiriart-Urruty. Global optimality conditions in maximizing a convex quadratic function under convex quadratic constraints. $J$. Global Optim., 21(4):445-455, 2001.

[Jar13] Bolor Jargalsaikhan. Indefinite copositive matrices with exactly one positive eigenvalue or exactly one negative eigenvalue. Electron. J. Linear Algebra, 26:754-761, 2013.

[JL13] Vaithilingam Jeyakumar and Guoyin Li. Trust-region problems with linear inequality constraints: exact SDP relaxation, global optimality and robust optimization. Math. Program., to appear. DOI:10.1007/s10107013-0716-2, 2013.

[JLL09] Vaithilingam Jeyakumar, Gue Myung Lee, and Guoyin Li. Alternative theorems for quadratic inequality systems and global quadratic optimization. SIAM J. Optim., 20(2):983-1001, 2009.

[LO13] Adrian S. Lewis and Michael L. Overton. Nonsmooth optimization via quasi-Newton methods. Math. Program., 141(1-2, Ser. A):135-163, 2013.

[Maj71] Antal Majthay. Optimality conditions for quadratic programming. Math. Program., 1:359-365, 1971.

[Mar94] José Mario Martínez. Local minimizers of quadratic functions on Euclidean balls and spheres. SIAM J. Optim., 4(1):159-176, 1994.

[MK87] Katta G. Murty and Santosh N. Kabadi. Some NP-complete problems in quadratic and nonlinear programming. Math. Program., 39(2):117-129, 1987. 
[NW06] Jorge Nocedal and Stephen J. Wright. Numerical Optimization. Springer Series in Operations Research and Financial Engineering. Springer, New York, second edition, 2006.

[NWY00] Yuri Nesterov, Henry Wolkowicz, and Yinyu Ye. Nonconvex quadratic optimization. In Henry Wolkowicz, Romesh Saigal, and Lieven Vandenberghe, editors, Handbook of Semidefinite Programming, pages 361-420. Kluwer, New York, 2000.

[PT07] Imre Pólik and Tamás Terlaky. A survey of the S-lemma. SIAM Rev., 49(3):371-418, 2007.

[PY97] Jiming Peng and Ya-Xiang Yuan. Optimality conditions for the minimization of a quadratic with two quadratic constraints. SIAM J. Optim., 7(3):579-594, 1997.

[YB13] Boshi Yang and Samuel Burer. A two-variable analysis of the two-trustregion subproblem. Preprint, submitted to SIAM J. Optim., http:// www.optimization-online.org/DB_HTML/2013/07/4126.html, 2013.

[Yua90] Ya-Xiang Yuan. On a subproblem of trust region algorithms for constrained optimization. Math. Program., 47(1, (Ser. A)):53-63, 1990.

[YZ03] Yinyu Ye and Shuzhong Zhang. New results on quadratic minimization. SIAM J. Optim., 14(1):245-267, 2003. 